\title{
From drawing to animation-ready vector graphics
}

\author{
E. Entem ${ }^{1,2}$ L. Barthe ${ }^{2}$ M.-P. Cani ${ }^{1}$ M. van de Panne ${ }^{3}$ \\ ${ }^{1}$ Université de Grenoble-Alpes, CNRS (Laboratoire Jean Kuntzmann) and Inria \\ ${ }^{2}$ Université de Toulouse, IRIT $\quad{ }^{3}$ University of British Columbia
}

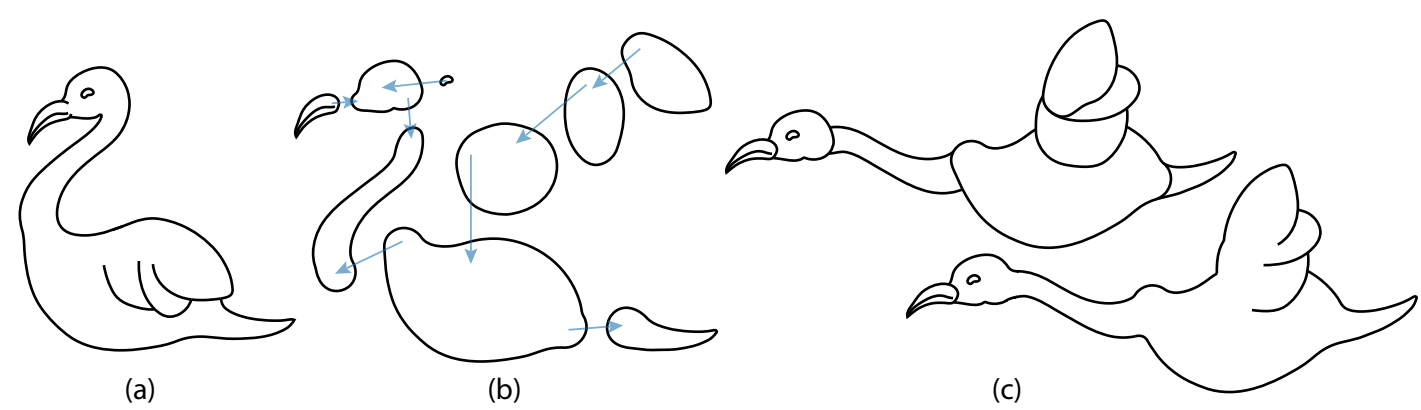

Figure 1: (a) An input clean-line vector drawing of a swan. It can be obtained using vectorization techniques on a raster image.

(b) Exploded view of the output. It consists of a layered structure of simpler and smoothly closed sub-regions corresponding to structural parts of the depicted 3D shape. Arrows represent the partial depth ordering ("over" relation). (c, top) The resulting structure can be used as a jumping-jack puppet for posing and animation purposes. (c, bottom) A frame of the animated drawing can be obtained by displaying the initially-suggestive parts of contours (inner curves) plus the new contour silhouette of the blended union of all the sub-regions.

\section{Abstract}

We present an automatic method to build a layered vector graphics structure ready for animation from a clean-line vector drawing of an organic, smooth shape. Inspiring from 3D segmentation methods, we introduce a new metric computed on the medial axis of a region to identify and quantify the visual salience of a sub-region relative to the rest. This enables us to recursively separate each region into two closed sub-regions at the location of the most salient junction. The resulting structure, layered in depth, can be used to pose and animate the drawing using a regular $2 \mathrm{D}$ skeleton.

Keywords: Vector Drawing analysis, Shape segmentation, Drawing edition

Concepts: -Computing methodologies $\rightarrow$ Image processing; Shape analysis;

\section{Introduction and Motivation}

Contour drawings are very commonly used for shape depiction. The fact that they are both easy to create and easy to interpret for a human makes them a convenient and expressive solution for visual communication. We can find those in children books, advertisements, technical books, and more. However they remain difficult to edit or manipulate. Despite their easy interpretation by humans, these drawings are particularly difficult to interpret for a computer, as they usually depict external and internal silhouettes as well as purely expressive features, and may represent fully visible, selfoccluding and/or partially hidden parts. Some algorithms have been

Permission to make digital or hard copies of part or all of this work for personal or classroom use is granted without fee provided that copies are not made or distributed for profit or commercial advantage and that copies bear this notice and the full citation on the first page. Copyrights for thirdparty components of this work must be honored. For all other uses, contact the owner/author(s). (C) 2016 Copyright held by the owner/author(s).

SIGGRAPH '16, July 24-28, 2016, Anaheim, CA,

ISBN: 978-1-4503-4371-8/16/07

DOI: http://dx.doi.org/10.1145/2945078.2945130 proposed to build 3D representations from Computer Aided Design sketches. Other methods tackle free-form drawings, but are generally dedicated to specific kinds of shapes. The technique presented in [Karpenko and Hughes 2006] handles smooth shapes, but their goal is 3D modeling and the intermediate depth and structural information is not suited for posing and animation purposes. We show a novel technique to structure clean-line drawings of smooth shapes into an manipulable representation.

\section{Our approach}

We propose a 2D part-aware metric to measure the relative salience along the medial axis of 2D shapes. Our metric is loosely inspired by the 3D metric in [Liu et al. 2009]. We cluster medial axis parts with high derivatives of this measure. We then define the area between each pair of contours corresponding to a cluster as a transition zone.

Processing possible transition zones between the identified subregions in a specific priority order enables us to achieve a recursive extraction of sub-regions, which handles the challenging case of multiple suggestive curves (inner trees of silhouette contours and of suggestive contours, connected to the silhouette contour of the processed region, as shown for the swan wing in Figure 1). The closure of identified sub-regions is performed by minimizing the curvature cost of the pair of closing cubic Bezier curves, with extremities and tangents being constrained by the type of transition zone (T-junction, suggestive contour, etc.). The relative depth between sub-regions is inferred from the information around $\mathrm{T}$-junctions.

The structure and layering information we obtain can be used to represent, edit and animate the input sketch in a meaningful way. It enables us to animate a drawing using a simple skeleton. In future work, we plan to compute the joints' positions automatically. In addition we could allow the interactive deformation of each part using medial-axis-based manipulators. 


\section{Acknowledgements}

This work was funded by the advanced grant EXPRESSIVE from the European Research Council (ERC-2011-ADG 20110209).

\section{References}

Karpenko, O. A., And Hughes, J. F. 2006. Smoothsketch: 3D free-form shapes from complex sketches. In ACM SIGGRAPH 2006 Papers, ACM, SIGGRAPH '06, 589-598.

LiU, R., Zhang, H., ShamiR, A., ANd COHEn-OR, D. 2009. A part-aware surface metric for shape analysis. Computer Graphics Forum (Special Issue of Eurographics) 28, 2, 397-406. 